\title{
Elementary remarks on units in monoidal categories
}

\author{
JOACHIM KOCK
}

\begin{abstract}
We explore an alternative definition of unit in a monoidal category originally due to Saavedra: a Saavedra unit is a cancellative idempotent (in a 1-categorical sense). This notion is more economical than the usual notion in terms of left-right constraints, and is motivated by higher category theory. To start, we describe the semi-monoidal category of all possible unit structures on a given semi-monoidal category and observe that it is contractible (if nonempty). Then we prove that the two notions of units are equivalent in a strong functorial sense. Next, it is shown that the unit compatibility condition for a (strong) monoidal functor is precisely the condition for the functor to lift to the categories of units, and it is explained how the notion of Saavedra unit naturally leads to the equivalent non-algebraic notion of fair monoidal category, where the contractible multitude of units is considered as a whole instead of choosing one unit. To finish, the lax version of the unit comparison is considered. The paper is self-contained. All arguments are elementary, some of them of a certain beauty.
\end{abstract}

Keywords: monoidal categories, units

Subject classification (MSC2000): 18D10

\section{Introduction}

Monoidal categories. Monoidal categories are everywhere in mathematics, and serve among other things as carrier for virtually all algebraic structures. Monoidal categories are also the simplest example of higher categories, being the same thing as bicategories with only one object, just as a monoid can be seen as a category with only one object. With the rapidly growing importance of higher category theory, it is interesting to revisit even the most basic theory of monoidal categories, to test new viewpoints and experiment with new formulations (cf. also Chapter 3 in Leinster's book [9]).

Units. This note analyses the notion of unit in monoidal categories. Units enjoy a mixed reputation: in some monoidal categories, the unit appears to be an insignificant part of the structure and is often swept under the carpet; in other cases (like in linear logic, in the theory of operads, or in higher categories), the proper treatment of units can be highly non-trivial, suggesting that we have not yet fully understood the nature of units. 
While the axioms for the multiplication law are rather well understood, and fit into a big pattern continuing in higher dimension (the geometrical insight provided by the Stasheff associahedra [12]), the unit axioms are subtler, and so far there seems to be no geometrical 'explanation' of them. This delicacy is perhaps also reflected historically. The first finite list of axioms for a monoidal category was given by Mac Lane [8] in 1963, including one axiom for associativity (the pentagon equation) and four axioms for the unit with its left and right constraints. Shortly after, it was shown by Kelly [6] that one of these four axioms for units in fact implies the three others. His proof constitutes nowadays the first three lemmas in many treatments of monoidal categories, while other sources continue to employ the redundant axiomatics. It is less well-known that conversely the three other unit axioms imply Kelly's axiom; this was observed by Saavedra [10] in 1972.

However, mere rearrangement of the axioms imposed on the unit structure is not the crux of the matter. The structure itself must be analysed. As it turns out, the classical notion of unit is overstructured. Saavedra [10] seems to have been the first to notice this: he observed that it is possible to express the notion of unit in monoidal categories without even mentioning the left and right constraints: a Saavedra unit (cf. [2.3) is an object $I$ equipped with an isomorphism $\alpha: I \otimes I \stackrel{\sim}{\rightarrow} I$, and having the property that tensoring with $I$ from either side is an equivalence of categories (in short, we say $I$ is a cancellable idempotent). Saavedra observed that this notion is equivalent to the classical notion (although his proof has a gap, as far as I can see, cf. Remark 2.14 below), but he did not pursue the investigation further - he did not even consider monoidal functors in this viewpoint.

The present note exploits the notion of Saavedra unit systematically to throw light also on the classical notion of unit and exhibit its redundancy. Many mathematicians experience this redundancy even at a naïve level, writing for example 'the right constraint is treated similarly', without realising that these phenomena can be distilled into precise results. At a deeper level, one may expect that the more economical notion of unit can help aliviate the nastiness of units felt at times, since in general it is cheaper to check a property than to provide a structure.

Terminology. Saavedra [10] used the term reduced unit for these cancellable idempotents, since they are less structured than the units-with-left-and-right-constraints. Another option would be absolute unit, referring to the fact that the notion makes sense prior to any associativity constraints, so for example it makes sense to fix the Saavedra unit and vary the associators. In contrast, the units axioms given in terms of left and right constraints make sense only relative to a fixed associativity constraint. But in any case it seems unfortunate to differentiate this alternative notion of unit from the usual notion by means of an extra adjective, since the former are not a special kind of the latter - the two notions are equivalent. From a purist's viewpoint, the cancellable-idempotent notion is what really should be 
called unit, the richer structure of left and right constraints being something derived, as explained in Section 2. In this note, a temporary terminology is adopted where both notions of unit carry an extra attribute: the notion of unit in terms of left-right constraints is called $L R$ unit, while the cancellable-idempotent notion is called Saavedra unit.

Overview of results. The material is organised as follows. In Section 1 after quickly reproducing Kelly's unit argument, we describe the category $U(\mathscr{C})$ of all possible LR unit structures on a semi-monoidal category $\mathscr{C}$ and show that it is contractible (if non-empty) (1.7). We observe that $U(\mathscr{C})$ has a canonical semimonoidal structure (1.8).

In Section 2]we introduce the notion of Saavedra unit, and show how Saavedra units are canonically LR units and vice versa (2.9). We also define morphisms of Saavedra units, and show that the category of LR units is isomorphic to the category of Saavedra units (2.19).

In Section 3 we study (strong) monoidal functors, and show that compatibility with LR units implies compatibility with Saavedra units and vice-versa (3.5), and more precisely (3.13): there is an isomorphism between the 2-category of monoidal categories with LR units (and strong monoidal functors and monoidal natural transformations) and the 2-category of monoidal categories with Saavedra units (and strong monoidal functors and monoidal natural transformations). Two corollaries are worth mentioning: first (3.12), a strong monoidal functor is compatible with left constraints if and only if it is compatible with right constraints, and in fact this compatibility can be measured on I alone! Second, a multiplicative functor is monoidal if and only if the image of a unit is again a unit (3.9). This statement does not even make sense for LR units (since the image of an LR unit does not have enough structure to make sense of the question whether it is a unit again). Finally we prove a rather technical result (3.17), needed in Section 5 a unit compatibility on a strong monoidal functor $\mathscr{C} \rightarrow \mathscr{D}$ is equivalent to a lift to the categories of units $U(\mathscr{C}) \rightarrow U(\mathscr{D})$.

Section 4 is a short interlude on monoids, placed here in order to motivate the next notion: in Section 5 we introduce a relativisation of the notion of cancellable object, here called gentle functors: these are functors $\mathscr{U} \rightarrow(\mathscr{C}, \otimes)$ such that the two composites $\mathscr{U} \times \mathscr{C} \rightarrow \mathscr{C} \times \mathscr{C} \stackrel{\otimes}{\rightarrow} \mathscr{C} \stackrel{\otimes}{\longleftarrow} \mathscr{C} \times \mathscr{C} \leftarrow \mathscr{C} \times \mathscr{U}$ are fully faithful. It is easy to see that a Saavedra unit in $\mathscr{C}$ is the same thing as a strong multiplicative functor $* \rightarrow \mathscr{C}$ which is furthermore gentle. Following this idea, we come to the notion of fair monoidal category: it is a gentle (strict) multiplicative functor $\mathscr{U} \rightarrow \mathscr{C}$ with $\mathscr{U}$ contractible. Here $\mathscr{U}$ is thought of as the category of all units in $\mathscr{C}$. It is shown (5.5) that this notion of monoidal category is equivalent to the classical notion (as claimed in [7]).

Finally, in Section 6 , we generalise some of the results about strong monoidal functors to lax monoidal functors. The Saavedra-unit compatibility is a bit less elegant to express in this case, but again we get an isomorphism of 2-categories 
(6.2). As a particular case we get an isomorphism of the categories of monoids in the LR and Saavedra-unit viewpoints, proving an assertion left open in Section 4,

Generalisations and outlook. Throughout we assume the tensor product to be strict. This is just for convenience: every argument can be carried over to the non-strict case, simply by inserting associator isomorphisms as needed. That complication would not seem to illuminate anything concerning units.

The notion of Saavedra unit has an obvious many-object version yielding an alternative notion of identity arrow in a bicategory. The notion of fair monoidal category described in Section 5 is just the one-object version of the notion of fair 2-category, which has generalisations to fair $n$-categories [7].

The remarks compiled in this note are a by-product of a more general investigation of weak units and weak identity arrows in higher categories [7], [4], [5], but as it turns out, even the 1-dimensional case contains some surprises, and I found it worthwhile to write it down separately and explicitly, since I think it deserves a broader audience. The notion of Saavedra unit dropped out of the theory of fair categories [7]. I am thankful to Georges Maltsiniotis for pointing out that the viewpoint goes back to Saavedra [10]. In this note, reversing my own path into the subject, it is shown how the basic notion of Saavedra unit leads to the notion of fair (monoidal) category. The two papers [4] and [5] joint with André Joyal deal with units in monoidal 2-categories. In [5] we use the 2dimensional notion of Saavedra unit to prove a version of Simpson's weak-unit conjecture [11] in dimension 3: strict 3-groupoids with weak units can model all 1-connected homotopy 3-types. The relevance of the Saavedra-unit viewpoint in higher-dimensional category theory was first suggested by Simpson [11]. The main advantage is its economical nature, and in particular it is important that the very notion of unit is expressed in terms of a multiplication map, already a central concept in the whole theory.

Acknowledgments. Part of this work was carried out while I was a postdoc at the Université du Québec à Montréal, supported by a CIRGET grant. I wish to thank everybody at the UQÀM, and André Joyal in particular, for a wonderful year in Montréal. Presently I am supported by a Ramón y Cajal fellowship from the Spanish Ministry of Science and Technology.

\section{The classical notion: LR units}

1.1 Semi-monoidal categories. A category with a multiplication, or a semi-monoidal category, is a category $\mathscr{C}$ equipped with an associative functor $\mathscr{C} \times \mathscr{C} \rightarrow \mathscr{C}$, here denoted by plain juxtaposition, $(X, Y) \mapsto X Y$. For simplicity we assume strict associativity, $X(Y Z)=(X Y) Z$. This is really no loss of generality: all the arguments in this note carry over to the case of non-strict associativity - just insert associators where needed. 
If $X$ is an object we use the same symbol $X$ for the identity arrow of $X$.

1.2 Monoidal categories. A semi-monoidal category $\mathscr{C}$ is a monoidal category when it is furthermore equipped with a distinguished object $I$, called the unit, and natural isomorphisms

$$
I X \stackrel{\lambda_{X}}{\longrightarrow} X \stackrel{\rho_{X}}{\longleftarrow} X I
$$

obeying the following rules (cf. Mac Lane [8]):

$$
\begin{gathered}
\lambda_{I}=\rho_{I} \\
\lambda_{X Y}=\lambda_{X} Y \\
\rho_{X Y}=X \rho_{Y} \\
X \lambda_{Y}=\rho_{X} Y
\end{gathered}
$$

It was observed by Kelly [6] that Axiom (4) implies the other three axioms. We quickly run through the arguments - they are really simple.

1.3 Naturality. Naturality of the left constraint $\lambda$ with respect to the arrow $\lambda_{X}$ : $I X \rightarrow X$ is expressed by this commutative diagram:

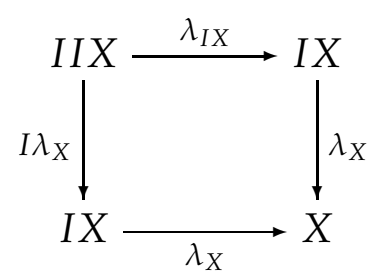

Since $\lambda_{X}$ is invertible, we conclude

$$
\lambda_{I X}=I \lambda_{X}
$$

Similarly with the right constraint:

$$
\rho_{X I}=\rho_{X} I .
$$

1.4 Fundamental observation. Tensoring with I from either side is an equivalence of categories:

$$
\begin{aligned}
& I \otimes_{-}: \mathscr{C} \simeq \mathscr{C}
\end{aligned}
$$

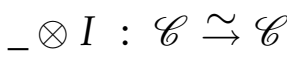

Indeed, the left and right constraints $\lambda$ and $\rho$ are invertible natural transformations between these two functors and the identity functors. This observation does not depend on the axioms (1)-(4). 
1.5 Lemma. (Kelly [6].) Axiom (4) implies axioms (1), (2), and (3).

Proof. (4) implies (2): Since tensoring with $I$ on the left is an equivalence, it is enough to prove $I \lambda_{X Y}=I \lambda_{X} Y$. But this equation follows from Axiom (4) applied twice (swap $\lambda$ out for a $\rho$ and swap back again only on the nearest factor):

$$
I \lambda_{X Y}=\rho_{I} X Y=I \lambda_{X} Y .
$$

Similarly for $\rho$, establishing (3).

(4) and (2) implies (1): Since tensoring with $I$ on the right is an equivalence, it is enough to prove $\lambda_{I} I=\rho_{I} I$. But this equation follows from (2), (5), and (4):

$$
\lambda_{I} I=\lambda_{I I}=I \lambda_{I}=\rho_{I} I .
$$

It was observed by Saavedra [10] that conversely (1), (2), and (3) imply (4). This will be an immediate corollary of the results in the next section, cf. 2.10,

1.6 The category of LR units A triple $(I, \lambda, \rho)$ as in 1.2 is called an $L R$ unit structure on $(\mathscr{C}, \otimes)$, or just an $L R$ unit. These form the objects of a category $U(\mathscr{C})$, the category of $L R$ units of $\mathscr{C}$, where an arrow $(I, \lambda, \rho) \rightarrow\left(I^{\prime}, \lambda^{\prime}, \rho^{\prime}\right)$ is given by an arrow $\psi: I \rightarrow I^{\prime}$ compatible with the left and right constraints in the sense that for every object $X$, these two triangles commute:
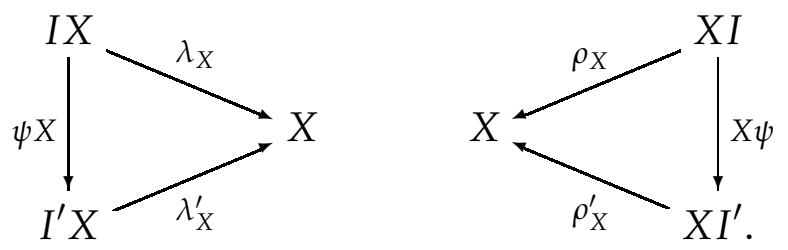

Note that the morphisms of LR units are precisely those with respect to which $\lambda$ and $\rho$ are also natural in $U$. That is, one can think of $\lambda$ (encoding all left constraints) as a natural transformation

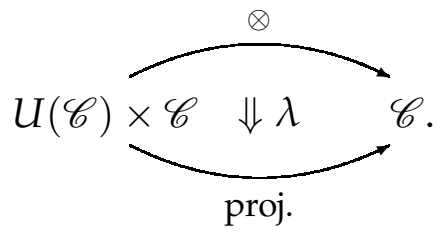

The component of $\lambda$ on $((I, \lambda, \rho), X)$ is simply $\lambda_{X}$.

It is a basic observation that for a semi-monoid the unit is unique if it exists. The following result, which goes back to Saavedra [10], shows that the LR unit for a monoidal category is unique up to unique isomorphism (if it exists).

1.7 Proposition. The category $U(\mathscr{C})$ is contractible. 
Proof. Given two LR units $(I, \lambda, \rho)$ and $\left(I^{\prime}, \lambda^{\prime}, \rho^{\prime}\right)$, define a map $\psi: I \rightarrow I^{\prime}$ by

$$
I \stackrel{\rho_{I}^{\prime-1}}{\longrightarrow} I I^{\prime} \stackrel{\lambda_{I^{\prime}}}{\longrightarrow} I^{\prime} .
$$

To see that $\psi$ is compatible with the left constraint as in (7), consider the diagram

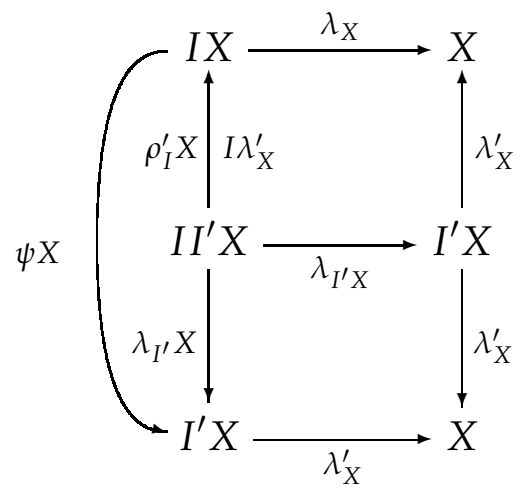

The bottom square is Equation (2) composed with $\lambda_{X}^{\prime}$. The top square is naturality of $\lambda$ with respect to $\lambda_{X}^{\prime}$. Since all the arrows are invertible, we can invert the vertical arrows of the top square; then the outline of the diagram is precisely the left-hand compatibility triangle in (7).

Compatibility with the right constraint is analogous to establish.

Finally we check that this $\psi$ is the only morphism of LR units from $I$ to $I^{\prime}$ : suppose we had another morphism of LR units $\gamma: I \rightarrow I^{\prime}$. Now take $X=I$ in the left-hand compatibility diagram (7). Since all the arrows involved are invertible, there can by at most one compatible arrow $I I \rightarrow I^{\prime} I$, so we have $\psi I=\gamma I$. But since tensoring with $I$ on the right is an equivalence, we conclude that $\psi=\gamma$.

1.8 Tensor product of LR units. There is a natural tensor on $U(\mathscr{C})$ : the tensor product of $(I, \lambda, \rho)$ with $\left(I^{\prime}, \lambda^{\prime}, \rho^{\prime}\right)$ is the object $I I^{\prime}$ equipped with left and right constraints given by these two composites:

$$
\begin{array}{ll}
I I^{\prime} X \stackrel{I \lambda_{X}^{\prime}}{\longrightarrow} I X \stackrel{\lambda_{X}}{\longrightarrow} X \\
X I I^{\prime} \stackrel{\rho_{X} I^{\prime}}{\longrightarrow} X I^{\prime} \stackrel{\rho_{X}^{\prime}}{\longrightarrow} X .
\end{array}
$$

It is easy to see that this tensor product is associative. It remains to check Axiom (4) for these new left and right constraints: in the diagram

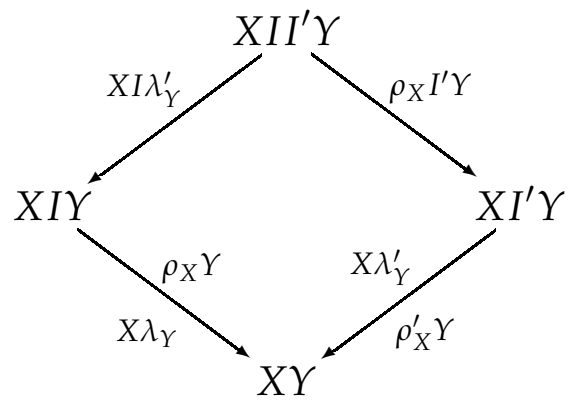


the inner labels show that the diagram commutes. The passage from inner labels to outer labels is just Axiom (4) for the original two units, and with the outer labels the diagram is precisely Axiom (4) for the new unit $I I^{\prime}$.

Note that the natural forgetful functor $U(\mathscr{C}) \rightarrow \mathscr{C}$ preserves the tensor product strictly.

We shall come back to this construction and show that the association $\mathscr{C} \mapsto$ $U(\mathscr{C})$ is functorial: a strong monoidal functor $\mathscr{C} \rightarrow \mathscr{D}$ induces a strong multiplicative functor $U(\mathscr{C}) \rightarrow U(\mathscr{D})$ compatible with the forgetful functors (Corollary 3.18 .

\section{Saavedra units}

2.1 Cancellable objects. An object $I$ in $\mathscr{C}$ is called cancellable if the two functors $\mathscr{C} \rightarrow \mathscr{C}$

$$
\begin{aligned}
& X \longmapsto I X \\
& X \longmapsto X I
\end{aligned}
$$

are fully faithful.

2.2 Idempotents. A idempotent in $\mathscr{C}$ is an object $I$ equipped with an isomorphism $\alpha: I I \rightarrow I$ which is associative, i.e., the equation $I \alpha=\alpha I$ holds. By a $p s e u d o-$ idempotent we mean the same thing but without requiring $\alpha$ to be associative.

2.3 Saavedra units. We define a Saavedra unit to be a cancellable pseudo-idempotent, and proceed to compare this notion with the notion of LR unit.

The two conditions in the definition of Saavedra unit strengthen each other mutually:

2.4 Lemma. (i) A Saavedra unit $(I, \alpha)$ is in fact an idempotent, i.e. $\alpha$ is automatically associative. In other words, $(I, \alpha)$ is a semi-monoid.

(ii) The two functors $\mathscr{C} \rightarrow \mathscr{C}$ given by $X \mapsto I X$ and $X \mapsto X I$ are in fact automatically equivalences.

This will be an easy consequence of the comparison with the LR notion of unit expressed by the next two lemmas.

2.5 Lemma. Given a Saavedra unit $(I, \alpha)$, for each object $X$ there are unique arrows

$$
I X \stackrel{\lambda_{X}}{\longrightarrow} X \stackrel{\rho_{X}}{\longleftarrow} X I
$$


such that

$$
\begin{gathered}
I \lambda_{X}=\alpha X \\
\rho_{X} I=X \alpha
\end{gathered}
$$

The $\lambda_{X}$ and $\rho_{X}$ are isomorphisms and natural in $X$.

These two equations will be invoked throughout.

Proof. Let $\mathbb{L}: \mathscr{C} \rightarrow \mathscr{C}$ denote the functor defined by tensoring with $I$ on the left. That $\mathbb{L}$ is fully faithful means we have a bijection on hom sets

$$
\mathscr{C}(I X, X) \rightarrow \mathscr{C}(I I X, I X) .
$$

Now take $\lambda_{X}$ to be the inverse image of $\alpha X$; it is an isomorphism since $\alpha X$ is. Naturality follows by considering more generally the bijection

$$
\operatorname{Nat}\left(\mathbb{L}, \operatorname{id}_{\mathscr{C}}\right) \rightarrow \operatorname{Nat}(\mathbb{L} \circ \mathbb{L}, \mathbb{L}) ;
$$

let $\lambda$ be the inverse image of the natural transformation whose components are $\alpha X$.

Similarly on the right.

2.6 Proposition. The $\lambda$ and $\rho$ constructed from $\alpha$ satisfy Axiom (4):

$$
\rho_{X} Y=X \lambda_{Y}
$$

Hence a Saavedra unit has a canonical LR unit structure.

Proof. In the commutative square

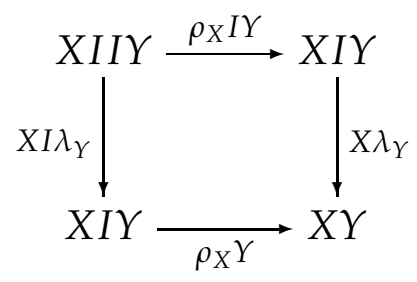

the left-hand arrow is equal to $X \alpha Y$, by $X$ tensor (8), and the top arrow is also equal to $X \alpha Y$, by (9) tensor $Y$. Since $X \alpha Y$ is an isomorphism, it follows that $\rho_{X} Y=$ $X \lambda_{Y}$.

Proof of 2.4 Re (i): set $X=Y=I$ in the Kelly equation (4), and apply (8) and (9) once again:

$$
I \alpha \stackrel{9}{=} \rho_{I} I=I \lambda_{I} \stackrel{8}{=} \alpha I .
$$

$\operatorname{Re}(\mathrm{ii)}: \mathbb{L}$ is an equivalence, because it is isomorphic to the identity via $\lambda$. (Similarly on the right.) 


\section{Conversely,}

2.7 Lemma. An LR unit $(I, \lambda, \rho)$ becomes a Saavedra unit by taking $\alpha:=\lambda_{I}=\rho_{I}$.

Indeed, $I$ is cancellable by 1.4

2.8 Back and forth. It is clear that starting from a Saavedra unit $(I, \alpha)$ and constructing $\lambda$ and $\rho$ as in 2.5, then $\lambda_{I}=\alpha$ and $\rho_{I}=\alpha$ (this follows from (10)). Conversely, starting from an $\operatorname{LR}$ unit $(I, \lambda, \rho)$ and setting $\alpha:=\lambda_{I}=\rho_{I}$, then we have

$$
\alpha X=\lambda_{I} X \stackrel{(2)}{=} \lambda_{I X} \stackrel{(5)}{=} I \lambda_{X}
$$

and similarly on the right, giving $X \alpha=\rho_{X} I$. Hence the original $\lambda$ and $\rho$ satisfy the equations defining the left and right constraints induced from $\alpha$. Altogether:

2.9 Proposition. There is a natural bijection between LR unit structures (on I) and Saavedra unit structures (on I).

We will see in a moment that this correspondence is functorial, with the appropriate notion of morphisms of Saavedra units. But first let us extract a few corollaries of the back-and-forth construction.

First, since the arguments in 2.7 do not depend on (4), we get the converse of Kelly's Observation 1.5 .

2.10 Corollary. Axioms (1), (2), and (3) together imply (4).

As noted above, (2) and (3) are needed to ensure that the back-and-forth construction gives back the original $\lambda$ and $\rho$. In absence of (2) and (3) we just get:

2.11 Corollary. Let $(\mathscr{C}, \otimes)$ be a semi-monoidal category and let I be an object equipped with natural isomorphisms $\lambda_{X}: I X \stackrel{\sim}{\rightarrow} X$ and $\rho_{X}: X I \stackrel{\sim}{\rightarrow} X$ such that $\lambda_{I}=\rho_{I}$, but not required to satisfy (2), (3), or (4). Then there exists an LR unit structure on I (possibly given by left and right constraints different from $\lambda$ and $\rho)$.

Indeed, put $\alpha=\lambda_{I}=\rho_{I}$, then clearly $(I, \alpha)$ is a Saavedra unit, and hence there are induced left and right constraints which satisfy (4), but there is no reason why these new left and right constraints should coincide with the original $\lambda$ and $\rho$.

Finally from the fact that $\lambda$ alone determines $\alpha$ (cf. 2.7), and $\alpha$ determines both $\lambda$ and $\rho$ (by 2.5), we get this:

2.12 Corollary. The left constraint and the right constraint of an LR unit determine each other. In other words, if $(I, \lambda, \rho)$ and $\left(I, \lambda, \rho^{\prime}\right)$ are both LR units then $\rho=\rho^{\prime}$ (and similarly with $\rho$ fixed). 
This corollary can also be deduced from contractibility of the category of units (the arguments in the proof of Proposition 1.7): the unique morphism of units between them is $\lambda_{I} \circ \rho_{I}^{-1}$, which is just the identity arrow of $I$, by (1). Now it follows from (7) that $\rho=\rho^{\prime}$.

2.13 Economy and absoluteness of Saavedra units. The LR notion of unit involves a lot of structure: two whole families of arrows are specified, and the Corollary reveals that this data is somewhat redundant. The notion of Saavedra unit is meant to be as economical as possible: the only structure to be specified is a single multiplication map $\alpha: I I \rightarrow I$, a notion already central to the theory of monoidal categories.

The notion of Saavedra unit is also more fundamental than the LR notion in that it is an absolute notion: namely, the notion of Saavedra unit makes sense prior to any associativity constraints, while in contrast the axioms for an LR unit only make sense relative to a specified associativity constraint. In this note, for simplicity, the associativity is assumed strict, and in particular fixed, so this remark does not play any role here. But as observed, all the arguments generalise to the nonstrict case by inserting associators when needed. Then it makes sense to fix the Saavedra unit and vary the associator; $\lambda$ and $\rho$ will then vary with the associator. (In the non-strict setting, the associator is involved in Construction 2.5] and the pentagon equation is required to establish Axiom (4) in Proposition 2.6).

2.14 Historical remarks. The notion of Saavedra units goes back to Saavedra's thesis [10] where it is mentioned in the preliminary chapter on monoidal categories as an alternative to the standard notion of unit. He doesn't really exploit the notion, though. He states the comparison result 2.9. but his proof (p.34-37) seems to contain an error. (First (Ch. I, 1.3), he defines a unit to be a triple $(I, \lambda, \rho)$ such that $\lambda_{I}=\rho_{I}$. Then he imposes conditions of compatibility with the associativity constraint (Ch. I, 2.2.1.1); these conditions are precisely (2), (3), and (4) above. He then proves (Ch. I, Prop. 2.2.3) the converse of Kelly's result, namely that (1)+(2)+(3) imply (4). Note that condition (1) can not be omitted. The definition of Saavedra unit is given in Ch. I, 1.3.2, and our Proposition 2.6 is part of his Proposition 2.2.5.1. The problem is this: after constructing $\lambda$ and $\rho$, he promises first to establish (2), (3), (4) before finally proving (1). However to prove (4) he uses Prop. 2.2.3 which crucially relies on (1) (and when he comes to proving (1) he uses (4)).)

2.15 Remark on strict units. An LR unit is strict if $\lambda_{X}$ and $\rho_{X}$ are identity arrows for all $X$. A Saavedra unit is strict if $\alpha$ is the identity arrow and if the two functors 'tensoring with $I$ ' are isomorphisms of categories. To see that the latter strictness implies the former, note that every object $X$ is of form $I Y$ for some $Y$. Now $\lambda_{I Y}=$ $\alpha Y$ is an identity arrow, so by naturality $\lambda_{X}$ is an identity arrow too. 
2.16 Tensor cancellable arrows. Let $I$ and $J$ be cancellable objects. An arrow $\psi: I \rightarrow J$ is called left tensor cancellable if the induced map on hom sets

$$
\begin{aligned}
\mathscr{C}(X, Y) & \longrightarrow \mathscr{C}(I X, J Y) \\
f & \longmapsto \psi f
\end{aligned}
$$

is a bijection. Right tensor cancellable is defined in the obvious way; an arrow is tensor cancellable if it is both left and right cancellable.

2.17 Morphisms of Saavedra units. Let $(I, \alpha)$ and $(J, \beta)$ be Saavedra units in $\mathscr{C}$. In partcular they are semi-monoids, by 2.4(i). A morphism of Saavedra units is a tensor cancellable semi-monoid homomorphism $\psi: I \rightarrow J$. Being a semi-monoid homomorphism means that this diagram commutes:

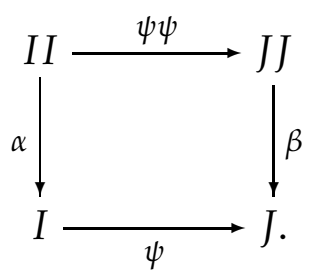

This defines the category of Saavedra units in $\mathscr{C}$.

2.18 Remark. By factoring (11) in two ways like this:

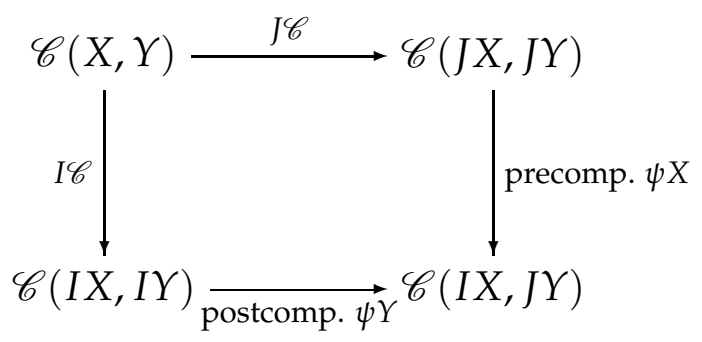

it follows that any monomorphism or epimorphism between cancellable objects is tensor cancellable. In particular, isomorphisms are tensor cancellable.

It follows from the next Proposition that a morphism of Saavedra units is automatically an isomorphism, since morphisms of LR units are so:

2.19 Proposition. The category of Saavedra units is canonically isomorphic to the category of LR units, hence contractible.

Proof. We have already established a bijection on the level of objects.

Given an arrow in the category of LR units, $\psi: I \rightarrow J$, then in particular it is an isomorphism and hence tensor cancellable. It remains to check that it is a 
semi-monoid homomorphism $(I, \alpha) \rightarrow(J, \beta)$. But this is easy: in the diagram

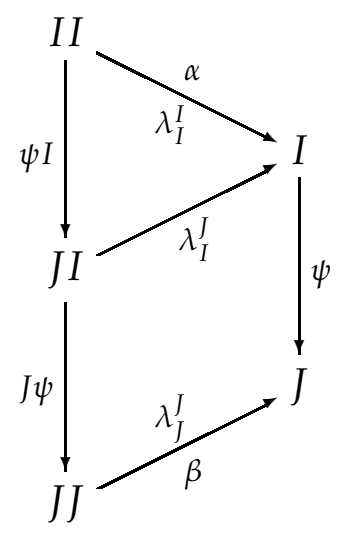

the triangle is compatibility (7) with the left constraint for the object $I$. The square is naturality of $\lambda^{J}$ with respect to $\psi$. The outer square is the semi-monoid homomorphism condition (12).

Conversely, suppose we have a tensor cancellable semi-monoid homomorphism $\psi: I \rightarrow J$. Construct $\lambda_{X}^{I}: I X \rightarrow X$ and $\lambda_{X}^{J}: J X \rightarrow X$ as in 2.5, and check that the composite

$$
\theta: \quad I X \stackrel{\psi X}{\longrightarrow} J X \stackrel{\lambda_{X}^{J}}{\longrightarrow} X
$$

is equal to $\lambda_{X}^{I}$ : In the diagram

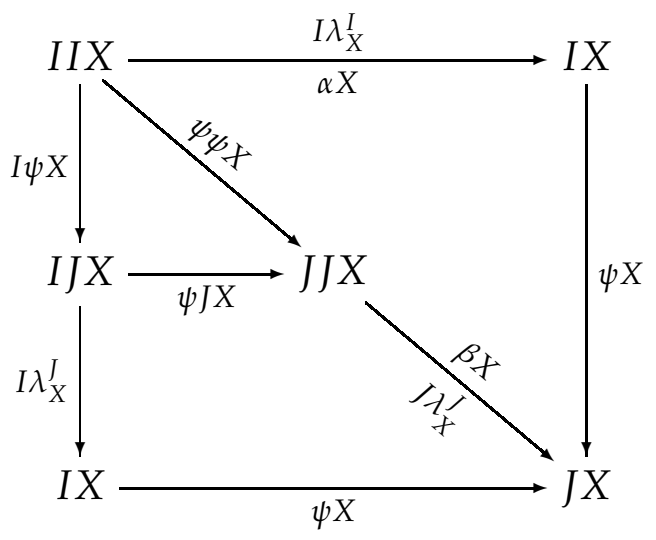

the upper right-hand cell is the semi-monoid homomorphism condition (12) (tensored with $X$ on the right), and the other cells are trivially commutative. Now the left-and-bottom composite is the tensor product $\psi \theta$ while the top-and-right composite is $\psi \lambda_{X}^{I}$. These two coincide since the outer square commutes. But since $\psi$ is (left) tensor cancellable, we conclude that $\theta=\lambda_{X}^{I}$ as wanted. A similar argument shows that $\psi$ is compatible with the right constraint.

Observe that in the passage from morphism of LR units to morphism of Saavedra units we only used compatibility with the left constraint (and could equally 
well have used only $\rho$ ), and in fact we only used the compatibility with respect to the object $X=I$. (It would also have been enough to test the compatibility with respect to the object $X=J$.) In particular:

2.20 Corollary. Given LR units $(I, \lambda, \rho)$ and $\left(I^{\prime}, \lambda^{\prime}, \rho^{\prime}\right)$, an arrow $I \rightarrow I^{\prime}$ is compatible with the left constraints if and only if it is compatible with the right constraints.

2.21 Tensor products of Saavedra units. The isomorphism of Proposition 2.19 endows the category of Saavedra units with a tensor product, which is surprising if you only look at the definition of Saavedra unit, where the only structure is a semi-monoid structure - usually in order to define tensor products of semimonoids you need a symmetry on the underlying semi-monoidal category.

Tracing through the correspondences, the tensor product of two Saavedra units $(I, \alpha)$ and $(J, \beta)$ is given by $(I J, \gamma)$ where $\gamma$ is the composite

$$
I J I J \stackrel{I J \lambda_{I}^{I}}{\longrightarrow} I J J \stackrel{\rho_{I}^{J} J}{\longrightarrow} I J .
$$

\section{Strong functoriality}

In this section we prove that the category of monoidal categories and strong monoidal functors in the LR-unit sense is isomorphic to the category of monoidal categories and strong monoidal functors in the Saavedra-unit sense.

3.1 Strong multiplicative functors. A strong multiplicative functor is a functor

$$
\begin{aligned}
& \mathscr{C} \longrightarrow \mathscr{D} \\
& X \longmapsto \bar{X}
\end{aligned}
$$

equipped with a natural family of isomorphisms in $\mathscr{D}$ :

$$
\phi_{2}:=\phi_{X, Y}: \bar{X} \bar{Y} \rightarrow \overline{X Y}
$$

such that this square commutes:

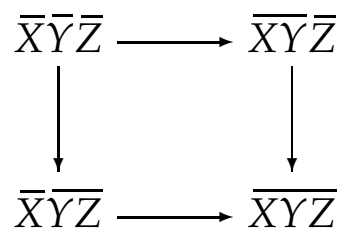

3.2 Strong monoidal functors in the LR-unit sense. A strong monoidal functor is a strong multiplicative functor $(\mathscr{C}, \otimes, I) \rightarrow(\mathscr{D}, \otimes, J), X \mapsto \bar{X}$ together with an 
isomorphism $\phi_{0}: J \rightarrow \bar{I}$ satisfying these two conditions of compatibility with the left and right constraints:
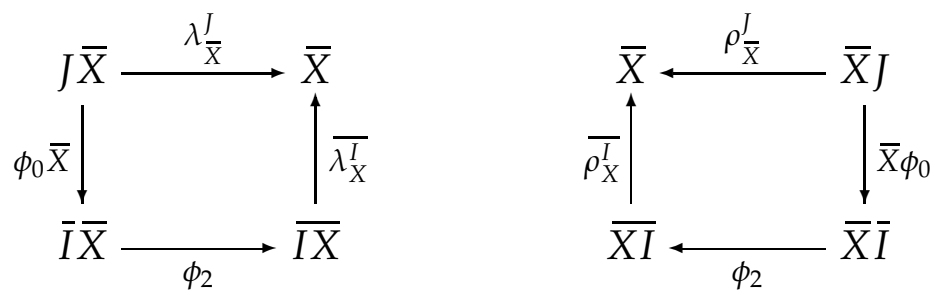

In Section 6 we shall consider also lax monoidal functors, where $\phi_{2}$ and $\phi_{0}$ are not required to be invertible.

3.3 Remark. Saavedra [10] considers only strong monoidal functors (and only in the LR-unit sense): he requires $\phi_{2}$ to be an isomorphism, and claims that then $\phi_{0}$ is automatically an isomorphism too ([10], Ch. I, 4.2.3.). This claim is false. It is true that $\phi_{0} \bar{X}$ is always an isomorphism, as seen in the diagram. But if $\bar{X}$ is not a cancellable object in $\mathscr{D}$, then this does not imply that $\phi_{0}$ is an isomorphism. As a concrete counter example, consider the lax monoidal functor Vect $\rightarrow$ Vect sending every vector space to the zero vector space. This is a strong multiplicative functor (in fact strict), but the unit comparison $\phi_{0}: \mathbb{k} \rightarrow \overline{\mathbb{k}}=\{0\}$ is obviously not an isomorphism. In the following paragraph, Saavedra states that a monoidal functor with a compatibility with given units might not have a compatibility with other units. This is correct as stated there, because at that point he does not assume Axiom (4) to hold. In that case the category of units is not necessarily connected, and compatibility is only guaranteed for units connected to the given compatible unit. When Axiom (4) is assumed to hold, as throughout the present note, compatibility with one unit implies compatibility with any other unit. This observation, and some related ones, will be made in the setting of Saavedra units where they are trivial to establish (cf. 3.8).

3.4 Strong monoidal functors in the Saavedra-unit sense. In the viewpoint of Saavedra units, a strong monoidal functor is defined to be a strong multiplicative functor $(\mathscr{C}, \otimes,(I, \alpha)) \rightarrow(\mathscr{D}, \otimes,(J, \beta))$ together with a semi-monoid isomorphism $\phi_{0}: J \rightarrow \bar{I}$.

Recall that $\bar{I}$ is a semi-monoid via the arrow

$$
\bar{I} \bar{I} \stackrel{\phi_{2}}{\longrightarrow} \overline{I I} \stackrel{\bar{\alpha}}{\longrightarrow} \bar{I},
$$


so the semi-monoid homomorphism condition is this:

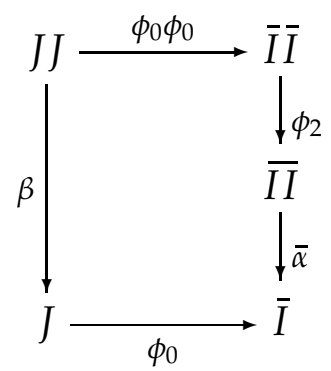

3.5 Proposition. The two compatibility conditions are equivalent. In other words, for a strong multiplicative functor, a compatibility with an LR unit canonically induces a compatibility with the corresponding Saavedra unit, and vice versa.

For later reference, we split the statement into two lemmas, Lemma 3.10 and Lemma 3.11 But first a couple of remarks, the first one being rather important:

3.6 Proposition. $\phi_{0}: J \rightarrow \bar{I}$ is a Saavedra-unit compatibility if and only if $(\bar{I}, \bar{\alpha})$ is a Saavedra unit and $\phi_{0}$ is a morphism of Saavedra units.

Here and below, by abuse of notation, the symbol $\bar{\alpha}$ refers to the composite (15).

Proof. Suppose $\phi_{0}$ is a unit compatibility, cf. 3.4 The fact that $\bar{I}$ is isomorphic to $J$ implies that it is itself cancellable, and hence $(\bar{I}, \bar{\alpha})$ is a Saavedra unit. The fact that $\phi_{0}$ is an invertible semi-monoid homomorphism is just to say that it is a morphism of units. Conversely, if $(\bar{I}, \bar{\alpha})$ is a Saavedra unit and $\phi_{0}$ is a morphism of units, then in particular it is an invertible semi-monoid homomorphism.

Note that this proposition can not be stated in terms of LR units, since a priori the image of an LR unit does not even have enough structure to make sense of the question whether it satisfies the unit axioms. (The left and right constraints of the image of the unit are only defined for objects in the image of the functor.)

Combining this proposition with contractibility of the category of units we get these three immediate corollaries:

3.7 Corollary. A unit compatibility on a strong multiplicative functor is unique if it exists.

This can be seen as a generalisation of the uniqueness part of Proposition 1.7 Namely, to give a morphism of units $I \rightarrow I^{\prime}$ in $\mathscr{C}$ is the same thing as providing a unit compatibility on the identity functor on $\mathscr{C}$, with $I$ chosen as unit in the domain and $I^{\prime}$ in the codomain.

3.8 Corollary. Let $\mathscr{C}$ and $\mathscr{D}$ be monoidal categories and let $\mathscr{C} \rightarrow \mathscr{D}$ be a strong multiplicative functor. Given a unit compatibility with respect to chosen units I in $\mathscr{C}$ and $J$ in $\mathscr{D}$, then there are canonical compatibilities with any other choices of units in $\mathscr{C}$ and $\mathscr{D}$. 
Finally, in view of Corollary 3.7 we can consider unit compatibility to be a property, not a structure, and restate Proposition 3.6 as:

3.9 Corollary. A multiplicative functor is monoidal if and only if the image of a unit is again a unit.

Again, this statement does not even make sense for LR units.

Now for the lemmas that make up Proposition 3.5

3.10 Lemma. If $\mathscr{C} \rightarrow \mathscr{D}, X \mapsto \bar{X}$ is a multiplicative functor, and $\phi_{0}: J \rightarrow \bar{I}$ is an LR-unit compatibility, then it is also a Saavedra-unit compatibility.

Proof. In fact it is enough to have the compatibility (14) with $\lambda$ for the object $X=I$. We then have a commutative diagram

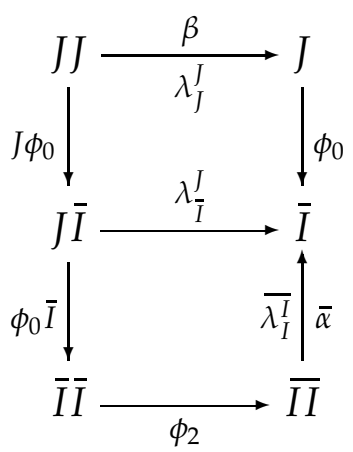

The top square is naturality of $\lambda^{J}$ with respect to $\phi_{0}$, and the bottom square is the left compatibility (14). The outer square is precisely the compatibility diagram (16) for $\phi_{0}$ with respect to the Saavedra units $\beta=\lambda_{J}^{J}$ and $\alpha=\lambda_{I}^{I}$.

3.11 Lemma. If $\phi_{0}: J \rightarrow \bar{I}$ is a Saavedra-unit compatibility then it is also compatible with $\lambda$ and $\rho$. 
Proof. In the diagram

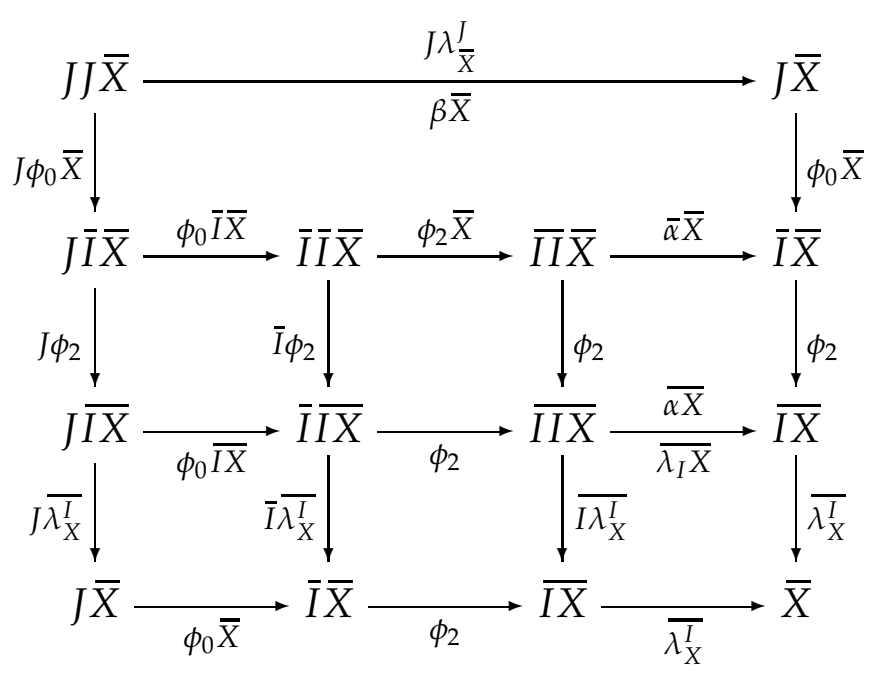

the top square is precisely the compatibility (16) between $\alpha$ and $\beta$ (tensored with $X)$; the other squares are commutative for trivial reasons. Now since the bottom composite and the right-hand composite in the big square are equal and are monomorphisms, we can cancel them away and conclude that the left-hand composite is equal to the top arrow as we wanted.

The compatibility with $\rho$ is established analogously.

In the proof of Lemma 3.10 we only used compatibility with $\lambda_{I}$, so as a corollary we get the following result, which does not refer to the notion of Saavedra unit.

3.12 Corollary. A strong monoidal functor (in the classical sense) is compatible with left constraints if and only if it is compatible with right constraints, and this can be measured on I alone.

Joining Proposition 3.5 and Proposition 2.9. and with the monoidal natural transformations as 2-cells (cf. just below), we get

3.13 Proposition. There is an isomorphism between the 2-category of monoidal categories, strong monoidal functors (in the LR sense), and monoidal natural transformations, and the 2-category of monoidal categories, strong monoidal functors in the Saavedra-unit sense, and monoidal natural transformations.

3.14 Monoidal natural transformations. A natural transformation $u:\left(F, \phi_{2}\right) \Rightarrow$ $\left(G, \gamma_{2}\right)$ between strong multiplicative functors $F: \mathscr{C} \rightarrow \mathscr{D}, X \mapsto \bar{X}$ and $G: \mathscr{C} \rightarrow$ $\mathscr{D}, X \mapsto \widetilde{X}$ is called multiplicative if for every pair of objects $X, Y$ in $\mathscr{C}$ this diagram 
commutes:

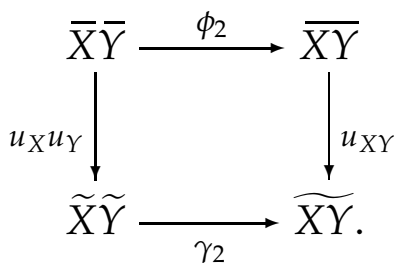

A multiplicative natural transformation is monoidal if the following unit condition is satisfied:

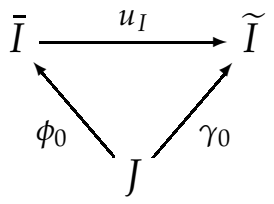

(It is the same condition for LR units and Saavedra units.)

There is one remark to make about monoidal natural transformations:

3.15 Lemma. Condition (18) holds automatically if $u_{I}$ is an isomorphism (or just tensor cancellable).

Proof. By $3.6,(\bar{I}, \bar{\alpha})$ and $(\widetilde{I}, \widetilde{\alpha})$ are Saavedra units, and $\phi_{0}$ and $\gamma_{0}$ are morphisms of units. Now, naturality of $u$ means that we have this commutative square

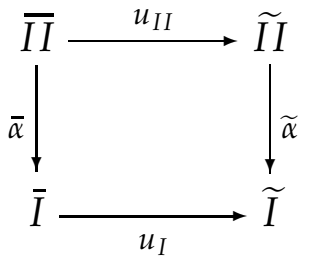

Hence if just $u_{I}$ is tensor cancellable then it is a morphism of units, and hence the triangle (18) commutes - every diagram of morphisms of units commutes because the category of units is contractible.

3.16 Unit compatibility in terms of multiplicativity for units. The following result is a variation of the corollaries 3.7 and 3.8 . but stated in a global manner involving also multiplicativity. It will play an important role in Section 5 .

3.17 Proposition. Let $\mathscr{C} \rightarrow \mathscr{D}$ be a multiplicative functor admitting a unit compatibility. The totality of all units compatibilities (one for each choice of unit in $\mathscr{C}$ and in $\mathscr{D})$ amounts precisely to a lift to a strong multiplicative functor $U(\mathscr{C}) \rightarrow U(\mathscr{D})$. I.e., a commutative diagram of strong multiplicative functors

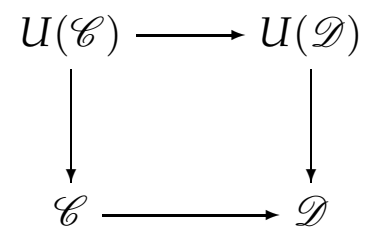


Proof. Given a lift, then for any $I$ in $U(\mathscr{C})$ and $J$ in $U(\mathscr{D})$, there is a connecting isomorphism $J \stackrel{\sim}{\rightarrow} \bar{I}$, since $U(\mathscr{D})$ is contractible. Hence by 3.6 we have a unit compatibility.

The converse implication is subtler. We already noticed (3.6) that the existence of a unit compatibility implies that the image of any unit is again a unit. It is also easy to see that if $\psi$ is a morphism of units then $\bar{\psi}$ is a morphism of units - this is just a consequence of the naturality of $\phi_{0}$ with respect to $\bar{\psi}$. Composition is obviously preserved; hence we have a functor $U(\mathscr{C}) \rightarrow U(\mathscr{D})$, and it is clear that diagram (19) commutes as a diagram of functors.

Furthermore, since $U(\mathscr{D})$ is contractible there exists a unique multiplicative structure on the functor $U(\mathscr{C}) \rightarrow U(\mathscr{D})$. Indeed, its components $\bar{I} \bar{I}^{\prime} \rightarrow \overline{I I^{\prime}}$ are the unique comparison arrows that exist in $U(\mathscr{D})$, and axiom (13) is automatically satisfied since all diagrams commute in a contractible category.

It remains to check that this map extends the $\phi_{2}$ of the original functor, i.e., that the diagram commutes as strong multiplicative functors, not just as functors. Verifying this amounts to checking that $\phi_{2}: \bar{I} \bar{I}^{\prime} \rightarrow \overline{I I^{\prime}}$ is a morphism of units, i.e. a semi-monoid homomorphism. Doing this in the Saavedra-unit setting is a bit cumbersome since the definition of the tensor products in the categories of units involve the left and right constraints anyway. But it is not difficult to check that $\phi_{2}: \bar{I} \bar{I}^{\prime} \rightarrow \overline{I I^{\prime}}$ is a morphism of LR units. We need to check that this diagram commutes for all objects $Y$ in $\mathscr{D}$ :

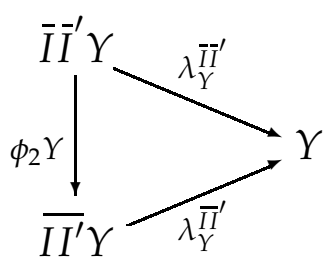

In view of Corollary 2.20 we do not have to check also the right-hand diagram, although of course this could be done analogously. By the remarks preceding that corollary, it is furthermore enough to check the diagram in the case $Y=\overline{I I^{\prime}}$. Since there are already so many Is involved, it is practical in the following argument to set $\bar{X}:=\overline{I I^{\prime}}$.

Note that for the unit $(\bar{I}, \bar{\alpha})$, the corresponding left constraint $\lambda_{\bar{X}}^{\bar{I}}$ is given by the equation

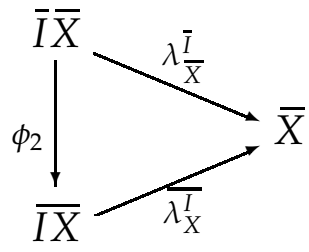

Now the wanted equation (20) is the outline of this big diagram: 


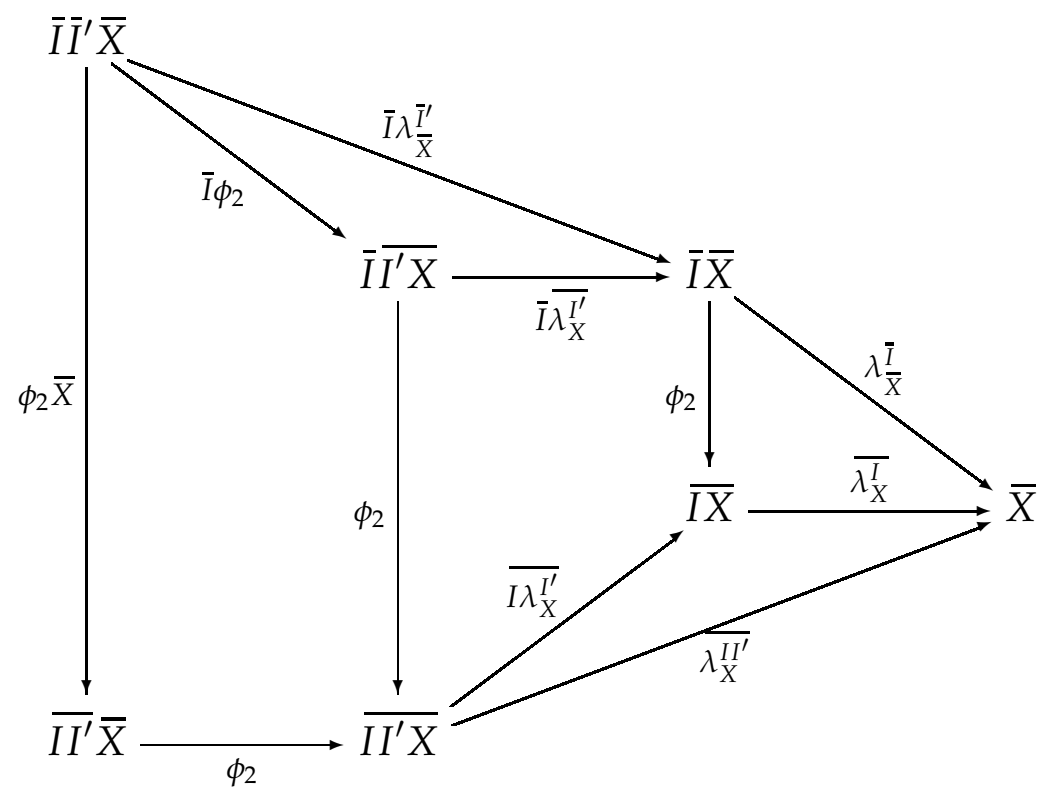

The left-hand square is associativity of $\phi_{2}$. The middle square is naturality of $\phi_{2}$. The three triangles are the definition of the left constraints as in (21). Now the top composite is precisely the left constraint of the tensor product $\bar{I} \bar{I}^{\prime}$ (by definition 1.8, and the bottom composite is the image of the left constraint on $I I^{\prime}$.

3.18 Corollary. The association $\mathscr{C} \rightarrow U(\mathscr{C})$ defines a functor from the category of monoidal categories and strong monoidal functors to the category of categories with multiplication and strong multiplicative functors.

\section{Monoids}

In this short section we describe what a monoid is in the Saavedra-unit setting. The proof is postponed to the end of the paper where it will be a special case of the treatment of lax monoidal functors. The Saavedra-unit notion of monoid motivates the fancy description of Saavedra units in Section 5 .

4.1 Semi-monoids. A semi-monoid in a semi-monoidal category $\mathscr{C}$ is an object $S$ equipped with an associative multiplication map $\mu: S S \rightarrow S$. A semi-monoid homomorphism from $(S, \mu)$ to $\left(S^{\prime}, \mu^{\prime}\right)$ is an arrow $S \rightarrow S^{\prime}$ such that this diagram commutes:

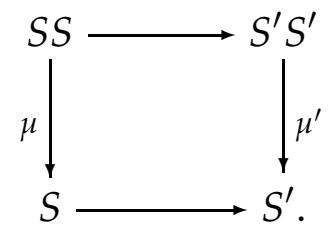


Henceforth when we refer to a monoidal category, we understand it to have both LR and Saavedra unit structure, corresponding to each other as described in Section 2 So a monoidal category is the data of $(\mathscr{C}, \otimes,(I, \alpha), \lambda, \rho)$.

4.2 Classical monoids. A monoid in the classical sense is a semi-monoid $\mu$ : $M M \rightarrow M$ equipped with an arrow $\eta: I \rightarrow M$ such that these two triangles commute:

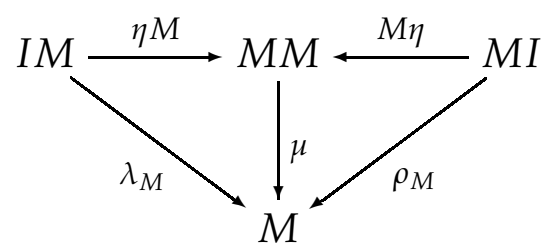

The arrow $\eta: I \rightarrow M$ with these properties is unique, if it exists.

4.3 Gentle maps. Let $(M, \mu)$ be a semi-monoid, and let $U$ be any object. We call an arrow $U \rightarrow M$ gentle if the two composites

$$
\begin{aligned}
& U M \rightarrow M M \stackrel{\mu}{\rightarrow} M \\
& M U \rightarrow M M \stackrel{\mu}{\rightarrow} M
\end{aligned}
$$

are monomorphisms.

4.4 Saavedra monoids. A Saavedra monoid is a semi-monoid $\mu: M M \rightarrow M$ equipped with a gentle semi-monoid homomorphism $\eta:(I, \alpha) \rightarrow(M, \mu)$ (i.e. a semi-monoid homomorphism that is gentle as an arrow in $\mathscr{C}$.)

As a consequence of the following proposition, in fact the two monomorphisms are automatically isomorphisms.

4.5 Proposition. There is an isomorphism between the category of classical monoids in $\mathscr{C}$ and the category of Saavedra monoids in $\mathscr{C}$.

The arrows in these categories are described in the following paragraph. The Proposition can be proved directly without difficulty, but we postpone the proof - it will be a special case of the treatment of lax monoidal functors, cf. page 28

4.6 Monoid homomorphisms. A monoid homomorphism between two monoids in $\mathscr{C}$ is just a semi-monoid homomorphism $\psi: M \rightarrow M^{\prime}$ such that this diagram commutes:

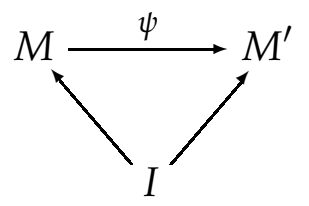

This is the same condition for Saavedra monoids. 


\section{Gentle multiplicative functors and fair monoidal categories}

In Section 4 we described a monoid in a monoidal category as a semi-monoid with a gentle semi-monoid homomorphism from the unit object. Clearly the notion of monoidal category should be a categorification of the notion of monoid, so let us reformulate the notion of Saavedra unit in terms of gentle multiplicative functors. This viewpoint also leads to the notion of fair monoidal category, which is the simplest manifestation of the higher-categorical concept of fair category, cf. [7].

Definition. We call a functor $\mathscr{U} \rightarrow(\mathscr{C}, \otimes)$ gentle if the two induced functors

$$
\begin{aligned}
& \mathscr{U} \times \mathscr{C} \longrightarrow \mathscr{C} \times \mathscr{C} \stackrel{\otimes}{\longrightarrow} \mathscr{C} \\
& \mathscr{C} \times \mathscr{U} \longrightarrow \mathscr{C} \times \mathscr{C} \stackrel{\otimes}{\longrightarrow} \mathscr{C}
\end{aligned}
$$

are fully faithful.

5.1 Lemma. Suppose $\mathscr{U}$ is contractible. Then $\mathscr{U} \rightarrow \mathscr{C}, U \mapsto \bar{U}$ is gentle if and only if $\bar{U}$ is cancellable for some (and hence for all) $U$ in $\mathscr{U}$.

Proof. We consider the left-hand conditions only. That $\mathscr{U} \rightarrow \mathscr{C}$ is gentle means that

$$
\mathscr{U}(I, J) \times \mathscr{C}(X, Y) \rightarrow \mathscr{C}(\bar{I} X, \bar{J} Y)
$$

is a bijection for all $I, J$ in $\mathscr{U}$ and all $X, Y$ in $\mathscr{C}$. But since $\mathscr{U}$ is contractible the set $\mathscr{U}(I, J)$ contains precisely one element $\psi$, so the bijection is just $\{\psi\} \times \mathscr{C}(X, Y) \rightarrow$ $\mathscr{C}(\bar{I} X, \bar{J} Y)$, given by tensoring with $\bar{\psi}$ on the left. This is to say that $\bar{\psi}$ is tensor cancellable. It remains to notice that since $\mathscr{U}$ is contractible, and since isomorphisms between cancellable objects are always tensor cancellable, $\bar{\psi}$ is tensor cancellable for all arrows $\psi$ in $\mathscr{U}$ if and only if $\bar{I}$ is cancellable for all objects $I$ in $\mathscr{U}$.

5.2 Corollary. A Saavedra unit in $\mathscr{C}$ is the same thing as a gentle, strong multiplicative functor $\eta: * \rightarrow \mathscr{C}$.

Proof. Call the image object $I$; this object is cancellable if and only if $\eta$ is gentle. Specifying the strong multiplicative compatibility on $\eta$ is equivalent to giving the isomorphism $\alpha: I I \rightarrow I$. (Note that since $I$ is cancellable, the multiplication map $\alpha$ is automatically associative by Lemma 2.4)

Similarly, a morphism of Saavedra units in $\mathscr{C}$ is the same thing as a multiplicative natural isomorphism between the corresponding functors $* \rightarrow \mathscr{C}$.

5.3 Fair monoidal categories. (Cf. [7].) The idea of fair monoidal category is to take the whole contractible category of units, instead of choosing an arbitrary unit in it. In the viewpoint of Corollary [5.2 a multiplicative category $\mathscr{C}$ is monoidal 
when it is equipped with a gentle, strong multiplicative functor $* \rightarrow \mathscr{C}$. By definition, a fair monoidal category is a gentle, strict multiplicative functor $\mathscr{U} \rightarrow \mathscr{C}$ between categories with multiplication $\mathscr{U}$ and $\mathscr{C}$, with $\mathscr{U}$ contractible.

Here $\mathscr{C}$ is thought of as the 'underlying category with multiplication', while $\mathscr{U}$ is thought of as its category of units. We want $\mathscr{U} \rightarrow \mathscr{C}$ to be strict because it should be thought of as the forgetful functor from the category of units.

5.4 Fair monoidal functors. Given fair monoidal categories $(U \rightarrow C)$ and $\left(U^{\prime} \rightarrow\right.$ $\left.C^{\prime}\right)$, a (strong) fair monoidal functor is a pair of strong multiplicative functors $\left(\phi_{U}, \phi_{C}\right)$ such that the diagram

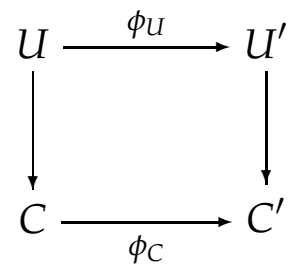

commutes (as strong multiplicative functors). (Note that in [7], only strict fair monoidal functors are considered, i.e. $\phi_{U}$ and $\phi_{C}$ are required to be strict multiplicative functors.)

5.5 Proposition. There is an equivalence of categories between the category MonCat of monoidal categories and strong monoidal functors and the category FairMonCat of fair monoidal categories and (strong) fair monoidal functors.

Proof. We describe first the functor MonCat $\rightarrow$ FairMonCat which is canonical, whereas the pseudo-inverse depends on a choice. Given a monoidal category $(\mathscr{C}, \otimes, I)$ then the category itself is a strict semi-monoidal category $C$. The category of units $U=U(\mathscr{C})$ is another strict semi-monoidal category, which is contractible, and there is a strict multiplicative functor $U \rightarrow C$, which is gentle by 5.1, since each unit is cancellable. Hence $U \rightarrow C$ is a fair monoidal category.

Given a multiplicative functor $(\mathscr{C}, \otimes, I) \rightarrow(\mathscr{D}, \otimes, J), X \mapsto \bar{X}$, the existence of a unit compatibility $\phi_{0}: J \stackrel{\sim}{\longrightarrow}$ means that $\bar{I}$ is a unit in $\mathscr{D}$ (by Proposition 3.6), and by Proposition 3.17, we can lift to a multiplicative functor $U(\mathscr{C}) \rightarrow U(\mathscr{D})$, by sending any unit $(I, \alpha)$ to $(\bar{I}, \bar{\alpha})$. (This is the only possible way to lift $\mathscr{C} \rightarrow \mathscr{D}$ to $U(\mathscr{C}) \rightarrow U(\mathscr{D})$.) By Proposition [3.17 the square (23) commutes as strong multiplicative functor, so we do have a fair monoidal functor.

Conversely, suppose we are given a fair monoidal category $(U \rightarrow C)$. Let $\mathscr{C}$ denote the category with multiplication $C$. We must provide it with a Saavedra unit. Just choose an object $I$ in $U$ - since $U$ is contractible, any choice is as good as any other choice. Now there is induced a canonical unit structure on its image $\bar{I}$ in $C$. Indeed, the fact that $U$ is contractible implies that there is a unique isomorphism $\alpha: I I \rightarrow I$, and the fact that $U \rightarrow C$ is gentle means that $\bar{I}$ is cancellable, by 5.1. Hence $\bar{I}$ is a Saavedra unit in $\mathscr{C}$. 
Suppose now we are given a diagram (23) of semi-monoidal categories, where $U$ and $U^{\prime}$ are contractible. We have already chosen $I \in U$ and $I^{\prime} \in U^{\prime}$ whose images are units in $C$ and $C^{\prime}$. The arrows $\alpha: I I \rightarrow I$ and $\alpha^{\prime}: I^{\prime} I^{\prime} \rightarrow I^{\prime}$ are given uniquely since $U$ and $U^{\prime}$ are contractible. Also since $U^{\prime}$ is contractible, there is a unique isomorphism $\phi_{0}: I^{\prime} \rightarrow \bar{I}$, and it is automatically compatible with $\bar{\alpha}$ and $\alpha^{\prime}$ because all compatibilities hold in a contractible category. The image of this compatibility diagram in $\mathscr{C}^{\prime}$ is the unit compatibility.

It is easy to see that the two constructions are pseudo-inverse to each other, in the sense that going back and forth gives something canonically isomorphic to the starting point, in either direction.

5.6 Monoids in fair monoidal categories. Monoids in fair monoidal categories have the following appealing description. A fair monoidal category is a gentle multiplicative functor $\eta: U \rightarrow C$ with $U$ contractible. A monoid in here is a gentle semi-monoid homomorphism $\eta(I) \rightarrow M$, where $I$ is a semi-monoid in $U$ and $M$ is a semi-monoid in $C$.

\section{Lax functoriality}

Many of the constructions and arguments of Section 3 admit a lax version, but not everything works as well in the lax case: the contractibility of the category of units makes it behave better under strong functors than under lax ones. For example, when $\phi_{0}: J \rightarrow \bar{I}$ is not invertible, $\bar{I}$ is not in general a unit itself.

The definition of lax monoidal functor in the LR setting is well-established: simply allow $\phi_{2}: \bar{X} \bar{Y} \rightarrow \overline{X Y}$ and $\phi_{0}: J \rightarrow \bar{I}$ to be non-invertible, and otherwise keep the conditions of 3.2 . One important motivation for considering lax monoidal functors is that monoids are a special case: a monoid in $\mathscr{C}$ is essentially the same as a lax monoidal functor $* \rightarrow \mathscr{C}$ (cf. Bénabou [2]).

6.1 Lax monoidal functors in the Saavedra-unit setting. A Saavedra-unit compatibility for a lax multiplicative functor $(\mathscr{C}, I) \rightarrow(\mathscr{D}, J), X \mapsto \bar{X}$ is a gentle semimonoid homomorphism $\phi_{0}: J \rightarrow \bar{I}$, such that

$$
\bar{I} \bar{X} \rightarrow \overline{I X} \text { and } \bar{X} \bar{I} \rightarrow \overline{X I} \text { are epi for all } X .
$$

Recall that gentle means that the two composites

$$
\begin{aligned}
& J \bar{I} \longrightarrow \bar{I} \bar{I} \longrightarrow \overline{I I} \longrightarrow \bar{I} \\
& \bar{I} J \longrightarrow \bar{I} \longrightarrow \bar{I} \longrightarrow \bar{I}
\end{aligned}
$$

are monomorphisms. (The following proposition implies that in fact those composites are isomorphisms.) 
6.2 Proposition. The two notions of lax monoidal functor coincide, under the correspondence of Proposition 2.9

The epi condition (24) may appear a little bit strange, and one might hope it would be unnecessary (i.e. automatically satisfied). Note that the LR unit compatibility of a lax monoidal functor implies that $\bar{I} \bar{X} \rightarrow \overline{I X}$ and $\bar{X} \bar{I} \rightarrow \overline{X I}$ are epi for all $X$. This follows from the conditions (14) since $\lambda_{X}$ and $\rho_{X}$ are isomorphisms.

Note that for strong monoidal functors, both condition (24) and (25) are automatic, so the definition reduces to 3.4 in this case.

Proof of Proposition 6.2 From LR unit compatibility to Saavedra unit compatibility works exactly like in the strong case (Lemma 3.10), noticing that the composites (25) are just $\lambda$ and $\rho$, and hence isomorphisms. We already observed that (24) is automatic for LR unit compatibilities.

The other direction, starting with a Saavedra-unit compatibility and showing that it is also an LR compatibility, is a little bit different from the strong case. First we show that the compatibility holds in the special case where the object is $I$. This is the content of Lemma 6.3. which does not use the epi condition (24). Then we use the epi condition to deduce the general result from this case (Lemma 6.4).

6.3 Lemma. The composite

$$
J \bar{I} \longrightarrow \bar{I} \bar{I} \longrightarrow \bar{I} \longrightarrow \bar{I}
$$

is precisely $\lambda_{\bar{I}^{J}}^{J}$. And similarly the other isomorphism is precisely $\rho_{\bar{I}}^{J}$. This does not depend on the epi condition (24).

Proof. We consider compatibility with $\lambda$; compatibility with $\rho$ is established analogously. By construction of $\lambda$, we have $J \lambda_{\bar{I}}^{J}=\beta \bar{I}$, so to establish the assertion of the lemma we tensor the composite with $J$ on the left and check that it gives $\beta \bar{I}$.

Consider the big diagram (17) of the proof in the strong case, but with $X=I$. In this case the right-hand composite and the bottom composite both coincide with (26), which is a monomorphism by assumption. So we can cancel them away and conclude that the lemma holds in the case $X=I$ as claimed.

6.4 Lemma. If $\phi_{0}: J \rightarrow \bar{I}$ is a Saavedra unit compatibility for a lax monoidal functor $X \mapsto \bar{X}$, then it is also an LR unit compatibility.

Proof. By the previous lemma, the LR compatibility holds for the object $I$. The next step is the case of an object of form IX: we claim that the composite

$$
\theta: J \overline{I X} \stackrel{\phi_{0} \overline{I X}}{\longrightarrow} \overline{I I X} \stackrel{\phi_{2}}{\longrightarrow} \overline{I I X} \stackrel{\overline{\lambda_{I X}^{I}}}{\longrightarrow} \overline{I X}
$$


is equal to $\lambda_{\overline{I X}}^{J}$. Tensor (26) with $\bar{X}$ on the right, and apply $\phi_{2}$ :

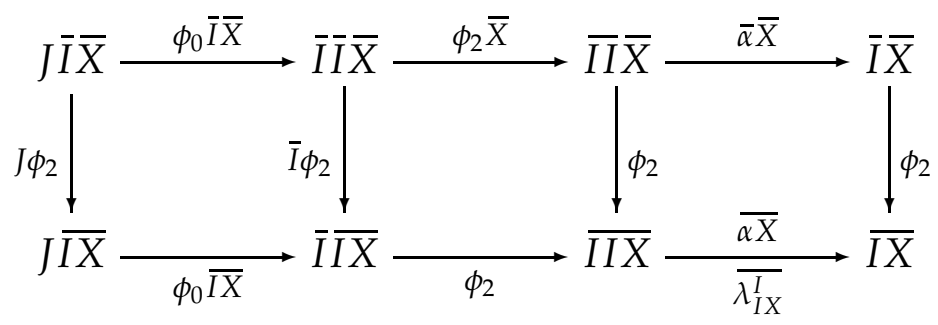

The first square is trivially commutative; the second is associativity of $\phi_{2}$ (cf. (13)); the third is naturality. The top row is $\lambda_{\bar{I}}^{J} \bar{X}=\lambda_{\bar{I} \bar{X}}^{J}$ (by basic property (2) of $\lambda$ ). But we also have the naturality square

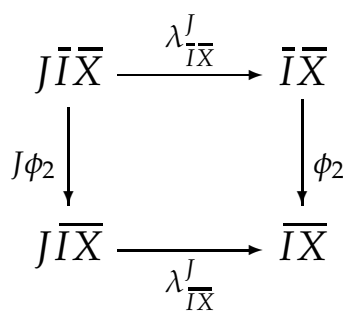

Hence the left-and-bottom ways around in these two diagrams coincide. Now $\phi_{2}$ is epi, and tensoring with $J$ on the left is an equivalence and hence preserves epimorphisms, so $J \phi_{2}$ is also epi. So we conclude that $\theta=\lambda_{\overline{I X}}^{J}$.

Now we have proved that the compatibility holds for every object of form IX:

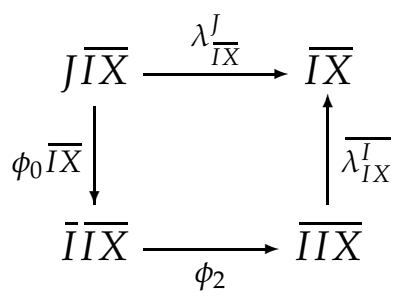

To finish the proof, use the isomorphism $\lambda_{X}^{I}: I X \stackrel{\sim}{\rightarrow}$ to compare with the diagram we want:

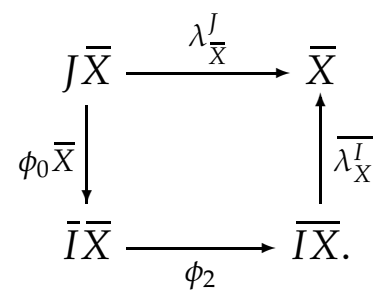

The compatibility with $\rho$ is established analogously.

6.5 Lemma. The epi condition (24) is automatically satisfied in the following cases: 
(a) $\phi_{0}$ is mono and $\phi_{I, X}$ and $\phi_{X, I}$ are mono for all $X$

(b) In the domain category, every object is isomorphic to $I$.

Proof. Re (a): If both $\phi_{0}$ and $\phi_{I, X}$ are mono, then the long composite

$$
J \bar{X} \rightarrow \bar{I} \bar{X} \rightarrow \overline{I X} \rightarrow \bar{X}
$$

is mono. But this was all we needed in the proof of Proposition 3.5 (proof of Lemma 3.11), so it carries over to the lax case.

$\operatorname{Re}($ b): Lemma 6.3 shows that $J \bar{I} \rightarrow \bar{I} \bar{I} \rightarrow \overline{I I} \rightarrow \bar{I}$ is an isomorphism independently of the epi condition (24). Hence $\bar{I} \bar{I} \rightarrow \overline{I I}$ is epi. But since $X$ is isomorphic to $I$, also $\bar{I} \bar{X} \rightarrow \overline{I X}$ is epi.

6.6 Monoids as lax monoidal functors. A monoid in $\mathscr{C}$ is essentially the same thing as a lax monoidal functor $* \rightarrow \mathscr{C}$. For the LR definition of lax monoidal functor this gives the classical notion of monoid, and for Saavedra-unit lax monoidal functors this gives the notion of Saavedra monoid of 4.4 This follows immediately since $\bar{*} \bar{*} \rightarrow \overline{* *}$ is automatically epi, cf. item (b) of the previous lemma.

Hence as a corollary we get Proposition 4.5

6.7 Remark. For strong monoidal functors we got Corollary 3.12 for free: compatibility with the left constraint implies compatibility with the right constraint. This result does not follow in the lax case, since we need both compatibilities (14) in order to be able to establish the epi condition (24). (The conclusion does hold in the special situations of 6.5) On the other hand, I don't know of a counter example to the statement in the lax case.

\section{References}

[1] Jean Bénabou. Catégories avec multiplication. C. R. Acad. Sci. Paris 256 (1963), 1887-1890.

[2] JEAN BÉNABOU. Algèbre élémentaire dans les catégories avec multiplication. C. R. Acad. Sci. Paris 258 (1964), 771-774.

[3] JEAn BÉNABOU. Introduction to bicategories. In Reports of the Midwest Category Seminar, pp. 1-77. LNM 47. Springer-Verlag, Berlin, 1967.

[4] André Joyal and JOACHIM KocK. Coherence for weak units. Manuscript in preparation.

[5] André JOYAL and JOACHIM KOCK. Weak units and homotopy 3-types. To appear in the StreetFest Proceedings (Contemp. Math., edited by M. Batanin, A. Davydov, M. Johnson, S. Lack, and A. Neeman). ArXiv:math.CT/0602084 
[6] G. MAX KeLly. On MacLane's conditions for coherence of natural associativities, commutativities, etc. J. Algebra 1 (1964), 397-402.

[7] JOACHIM KосK. Weak identity arrows in higher categories. Intern. Math. Res. Papers 2006 (2006), 1-54. (ArXiv:math.CT/0507116).

[8] SAUnders MaC LANE. Natural associativity and commutativity. Rice Univ. Studies 49 (1963), 28-46.

[9] TOM Leinster. Higher Operads, Higher Categories. Vol. 298 of London Math. Soc. Lecture Note Series. Cambridge University Press, Cambridge, 2004. (ArXiv:math.CT/0305049).

[10] Neantro SaAVedra Rivano. Catégories Tannakiennes. LNM 265. SpringerVerlag, Berlin Heidelberg, 1972.

[11] Carlos Simpson. Homotopy types of strict 3-groupoids. Preprint, ArXiv:math.CT/9810059

[12] James Dillon StashefF. Homotopy associativity of H-spaces. I, II. Trans. Amer. Math. Soc. 108 (1963), 275-292; 293-312.

ADDRESS: Departament de Matemàtiques, Universitat Autònoma de Barcelona, 08193 Bellaterra (Barcelona), Spain.

EMAIL ADDRESS: kock@mat.uab.cat 